\title{
Conocimiento para la enseñanza del número en futuras educadoras de párvulos: Efecto de un curso de didáctica de la matemática*
}

\author{
Knowledge for teaching number in preschool preservice teachers: The effect of a didactic \\ of mathematics course
}

Conhecimento para ensino do número em futuras educadoras da educação infantil: Resultado de um curso de didática da matemática

\section{Tatiana Goldrine, Soledad Estrella, Raimundo Olfos, Pablo Cáceres, Ximena Galdames, Natalia Hernández, Valeska Medina. ${ }^{a}$}

a Pontificia Universidad Católica de Valparaíso, Chile. Fono: 32-2274344.

Correo electrónico: tatiana.goldrine@ucv.cl

\begin{abstract}
RESUMEN
La investigación en formación docente inicial ha evidenciado en los futuros profesores conocimientos insuficientes para la enseñanza. En este contexto, se investigó el efecto de un curso de didáctica de la matemática en el conocimiento de futuras educadoras de párvulos para la enseñanza del número. El curso se basó en un constructo sobre el conocimiento docente que incluye el conocimiento de la educadora sobre lógica, número, etapas del aprendizaje del niño y organización de la enseñanza. Se utilizaron metodologías que integran teoría-práctica en la formación inicial, como el análisis de videos, el estudio de caso y el estudio de clase. Se usó un enfoque mixto, un diseño cuasi experimental con un test de conocimientos antes y después del curso, mapas conceptuales y entrevistas, constatándose diferencias significativas a favor del curso. El estudio provee un marco conceptual y metodologías para la formación docente en didáctica de la matemática en Educación Parvularia.

Palabras clave: educación parvularia, formación inicial docente, conocimiento del profesor, educación matemática, conocimiento pedagógico del contenido.
\end{abstract}

\begin{abstract}
Research on initial teacher education has evidenced insufficient prospective teachers knowledge about teaching. In this context, we investigated the effect of a mathematics education course in the knowledge of prospective preschool educators for the teaching of number. The course was based on a construct of teacher knowledge, including knowledge on logic, number, stage of children's learning and teaching organization. Methodologies as video analysis, case study and lesson study were used to integrate theory and practice in initial education. We used a mixed approach, quasi-experimental design with a test of knowledge before and after the course, concept maps and interviews, being stated significant differences in favor of the course. The study provides a conceptual framework and methodologies for initial training in didactics of mathematics for Early Childhood Education.
\end{abstract}

Key words: preschool education, initial teacher training, teacher knowledge, mathematics education, pedagogical content knowledge.

Esta investigación ha sido posible gracias al proyecto Fondecyt 1111009, financiado por la Comisión Nacional de Investigación Científica y Tecnológica (CONICYT) de Chile. 


\begin{abstract}
RESUMO
A pesquisa sobre formação inicial de professores tem evidenciado em futuros professores conhecimentos insuficientes para o ensino. Neste contexto, investiga-se o resultado de um curso de didática da matemática no conhecimento de futuras educadoras da Educação Infantil para o ensino do o número. O curso foi baseado na construção sobre o conhecimento dos professores, incluindo o conhecimento da educadora sobre lógica, número, etapas da aprendizagem das crianças e organização do ensino. Utilizaram-se metodologias que integram teoria e prática na formação inicial, como a análise de vídeos, o estudo de caso e estudo de aula. Utilizou-se um enfoque misto, um projeto quase experimental com um teste de conhecimentos antes e após o curso, mapas conceituais e entrevistas, constatando-se diferenças significativas em favor do curso. $\mathrm{O}$ estudo fornece um marco conceitual e metodologias para a formação de professores em didática da matemática na Educação Infantil.
\end{abstract}

Palavras chave: educação infantil, formação inicial de professores, conhecimento de professor, educação matemática, conhecimento pedagógico do conteúdo.

\title{
1. INTRODUCCIÓN
}

¿Qué tienen que saber los futuros profesores para enseñar? y ¿cómo transforman el conocimiento en prácticas de aula que beneficien el aprendizaje de los niños? son preguntas que motivan la investigación desde hace años (Ávalos, 2011; Hammerness et al., 2005). El desarrollo profesional docente es un componente crítico en la educación infantil, y es un factor que predice resultados de aprendizaje (Saracho \& Spodek, 2007).

Sin embargo, investigaciones recientes reportan en educadoras de párvulos en servicio y en formación inicial conocimientos insuficientes para la enseñanza. Una investigación sobre el conocimiento para la enseñanza de la matemática en futuras docentes de Educación Parvularia desarrollado en Turquía encontró conocimientos insuficientes sobre los conceptos matemáticos trabajados en educación infantil (Esen, Özgeldi \& Haser, 2012). Un estudio norteamericano constató baja coincidencia entre lo que las docentes de infantil reportan como importante en el aprendizaje de la matemática y lo observado en sus clases (Varol, Farran, Bilbrey, Vorhaus \& Hofer, 2012).

Estudios con docentes chilenas de Educación Parvularia en servicio evidencian falencias en el dominio conceptual y una falta de intencionalidad educativa para asumir el proceso pedagógico (Morales, Quilaqueo y Uribe, 2010), distanciamiento entre el lenguaje matemático informal y el lenguaje disciplinar, y predominio del conocimiento cotidiano por sobre el didáctico (Friz, Sanhueza, Sánchez, Sámuel y Carrera, 2009; Friz, Sanhueza y Samuel, 2008). En el contexto nacional, los resultados de la Prueba Inicia muestran que las estudiantes de las carreras de Educación Parvularia al egreso de la formación dominan alrededor de un 50\% de los conocimientos estimados para la enseñanza (MINEDUC, 2012, 2011, 2010).

La distinción entre el conocimiento del profesor para enseñar un dominio específico y el conocimiento de ese dominio llevó a Shulman (1987) a identificar tres componentes del conocimiento requerido para la enseñanza: conocimiento del contenido (CC), conocimiento pedagógico (CP) y conocimiento pedagógico del contenido (CPC). Desarrollos posteriores sobre el CC y CPC (McCray \& Chen, 2012; Lee, 2010; Krauss, Baumert \& Blum, 2008; Krauss, Brunner \& Kunter, 2008; Hill, Ball \& Schilling, 2008; McCray, 2008; Platas, 2008; Fennema \& Franke, 1992) y diversas investigaciones que avalan al CC y CPC del profesor como factores relevantes en la efectividad de la enseñanza de la matemática (Schmidt et al., 2007; Sliva, Strage \& Bergthold, 2007; Ball, Lubienski \& Mewborn, 2001; Ma, 1999; Fennema \& Franke, 1992) aportan sustento para conceptualizar el conocimiento de la educadora de párvulos para la enseñanza de la matemática. 
El conocimiento docente para la enseñanza de la matemática está ligado a la enseñanza de objetos matemáticos específicos. En particular, el número es fundamental en el desarrollo del pensamiento matemático en los primeros años, por lo que resulta relevante estudiar el conocimiento del docente para la enseñanza del número en el nivel de Educación Parvularia.

Lee (2010) investigó el CPC para la enseñanza de la matemática en docentes de Educación Infantil, encontrando que la noción de mayor preponderancia es el sentido del número, seguido de las nociones de patrones, seriación, formas, comparación y espacio. Además encontró correlación entre el CPC con los años de experiencia y de formación profesional, sosteniendo que en la formación inicial docente se requiere una formación específica en enseñanza de la matemática.

Platas (2008) midió el conocimiento docente sobre el desarrollo matemático de los niños, focalizando la indagación en el ámbito de números y operaciones por ser el más relevante en la educación matemática infantil. Al igual que Lee (2010), encontró que las docentes con más años de experiencia y con formación general y específica en desarrollo matemático infantil presentan mayor conocimiento para la enseñanza. Platas (2008) sugiere que las educadoras desarrollen: i) conocimiento de los conceptos matemáticos propios de este nivel educativo; ii) conocimiento pedagógico del contenido que incluya una profunda comprensión del desarrollo del pensamiento lógico-matemático infantil, y; iii) de las representaciones y estrategias apropiadas para la construcción de los conceptos matemáticos. De lo anterior se deriva que en la formación docente inicial es relevante que las futuras educadoras comprendan la matemática a enseñar y los procesos de construcción de conocimientos matemáticos en los niños, a fin de clarificar qué enseñar y cómo enseñar en este nivel educativo (McCray \& Chen, 2012; McCray, 2008).

En base a los referentes anteriores, los autores del presente trabajo elaboraron un constructo sobre el conocimiento docente para la enseñanza del número compuesto por CC, CPC-Ens y CPC-CRAC (Goldrine, Estrella, Olfos y Cáceres, en revisión). El CC abarca el conocimiento docente sobre los conceptos de lógica y número, y las representaciones semióticas idóneas de tales nociones para su enseñanza en este nivel. El CPC contiene dos componentes, una acerca de la Enseñanza del número, CPC-Ens, que incluye el conocimiento docente sobre la secuencia de tareas matemáticas para la enseñanza de la lógica y del número, conocimiento del currículo oficial de este nivel educativo, conocimiento de materiales para la representación de nociones lógico matemáticas y creencias docentes sobre la enseñanza y el aprendizaje. La otra componente considera el Conocimiento Docente sobre la Relación de los Alumnos con el Contenido, CPC-CRAC, que incluye el conocimiento docente sobre etapas en el aprendizaje de nociones de lógica y número, como, por ejemplo, etapas de dominio de la serie oral, etapas en la simbolización de cantidades, además del conocimiento docente acerca de los errores frecuentes de los párvulos, por ejemplo, errores en el conteo.

Por otra parte, con respecto a la formación inicial docente, tradicionalmente los modelos de formación han privilegiado la transmisión de conocimientos disciplinarios y pedagógicos. Un estudio que revisó modelos innovadores en formación del profesorado identificó como características relevantes la relación dialéctica entre teoría y práctica, y la presencia de la reflexión en la formación docente (Murillo, 2006). Perrenoud (2004) postula que la formación docente debe favorecer la capacidad para reflexionar sobre la práctica, propiciando que el futuro profesor transite desde un rol pasivo de alumno hacia un rol activo en su propia formación. La reflexión sobre la práctica favorece la articulación 
de los conocimientos con los saberes de la experiencia, la innovación pedagógica y la profesionalización docente. Atendiendo estas premisas, esta investigación utiliza el análisis de videos de prácticas de aula, el estudio de clases y el estudio de casos, como experiencias formativas que integran teoría con práctica y reflexión docente en la formación de futuras educadoras de párvulos.

La literatura actual muestra la incorporación del análisis de videos, el estudio de clases y el estudio de casos como metodologías para la formación del profesorado. Brophy (2004) señala el auge del uso de clases videograbadas en la formación docente. En particular, el análisis de videos propios muestra gran potencial para activar los conocimientos y la visión profesional (Seidel, Stürmer, Blomberg, Kobarg, \& Schwindt, 2011). El análisis de videos entre pares aparece en la literatura como una herramienta para fomentar discusiones productivas sobre la enseñanza y el aprendizaje de la matemática (Koc, Peker \& Osmanoglu, 2009; Borko, Jacobs, Eiteljorg \& Pittman, 2008; Harford \& MacRuairc, 2008; Santagata, Zannoni \& Stigler, 2007; Lundeberg, Levin \& Harrington, 1999; Darling-Hammond, 1997; Hiebert et al., 1997).

Isoda, Arcavi y Mena (2007) señalan que el estudio de clases es una estrategia de desarrollo profesional docente creada en Japón hace 140 años, actualmente expandida en más de una veintena de países. El estudio de clases consiste en la preparación, implementación, observación y discusión de una clase por un grupo de profesores. Investigaciones reportan que el estudio de clases es una herramienta que los profesores valoran porque propicia la identificación de los atributos de la clase (Lee, 2008; Puchner \& Taylor, 2006).

El estudio de casos data de la década de 1920 en la preparación de profesionales en áreas que requieren de una articulación entre la formación teórica y práctica. Consiste en el análisis y el diálogo entre futuros profesionales frente a una situación problema, lo que permite relacionar dialécticamente teoría y práctica en un proceso reflexivo que moviliza conocimientos y concepciones dentro del ámbito de desempeño profesional. Actualmente, en Chile, ha sido utilizado en la formación de profesores de matemáticas (Reyes, 2011), a partir de la presentación de una situación ficticia de aula que plantea conflictos pedagógicos y didácticos a los cuales el futuro profesor es enfrentado para hacer emerger sus conocimientos y reflexión docente.

\subsection{CURSO DE DIDÁCTICA DE LA MATEMÁTICA}

En el contexto de la búsqueda de estrategias para mejorar la formación inicial de educadoras de párvulos, este estudio diseñó un curso de didáctica de la matemática basado en el constructo conocimiento docente para la enseñanza del número y en las tres metodologías de enseñanza expuestas. El curso constó de 16 sesiones, incluidas una sesión de pretest y otra de postest. Los contenidos (ver Tabla $\mathrm{N}^{\circ} 1$ ) abordaron el desarrollo del número en el niño, conceptos de didáctica de la matemática (análisis a priori, situación problema, devolución, institucionalización y variables didácticas, entre otros), y el enfoque de resolución de problemas (Isoda y Olfos, 2009). La metodología del curso incluyó exposiciones de contenidos sobre lógica y número, actividades de aplicación y lecturas complementarias, además del estudio de clases, estudio de casos y el análisis de videos de futuras docentes con párvulos en aulas reales. En el curso, las estudiantes diseñaron una experiencia de enseñanza para el aprendizaje del número, que implementaron con un grupo de párvulos y videograbaron para su posterior análisis. 
Tabla $N^{\circ} 1$. Curso de didáctica de la matemática para enseñanza del número en Educación Parvularia

\begin{tabular}{|l|l|}
\hline \multicolumn{1}{|c|}{$\mathbf{N}^{\text {0 Sesión/ Contenidos }}$} & \multicolumn{1}{|c|}{ Metodología } \\
\hline $\begin{array}{l}\text { S1/Enfoque de resolución de } \\
\text { problemas en Matemática. }\end{array}$ & $\begin{array}{l}\text { Análisis de video. Planificación de una experiencia de aprendizaje } \\
\text { para párvulos sobre el concepto de número a partir de los } \\
\text { conocimientos docentes previos. }\end{array}$ \\
\hline $\begin{array}{l}\text { S2/Resolución de problemas } \\
\text { en Educación Parvularia. }\end{array}$ & $\begin{array}{l}\text { Re-elaboración de experiencia de aprendizaje para párvulos sobre } \\
\text { el número incorporando una situación problema. }\end{array}$ \\
\hline S3/Iniciación a la Lógica. & Análisis de video. Lecturas complementarias. \\
\hline S4/Iniciación a la Lógica. & $\begin{array}{l}\text { Elaboración de experiencia para párvulos sobre la lógica } \\
\text { incorporando una situación problema. }\end{array}$ \\
\hline S5/Construcción del Número. & Análisis de video. Lecturas complementarias. \\
\hline $\begin{array}{l}\text { S6/Componentes del Análisis } \\
\text { A Priori. }\end{array}$ & $\begin{array}{l}\text { Análisis de video. Análisis a priori de experiencia planificada para } \\
\text { párvulos sobre el número. }\end{array}$ \\
\hline $\begin{array}{l}\text { S7/Resolución de problemas } \\
\text { en Educación Parvularia. }\end{array}$ & $\begin{array}{l}\text { Re-elaboración de experiencia para párvulos sobre el número } \\
\text { incorporando el enfoque de resolución de problemas. }\end{array}$ \\
\hline $\begin{array}{l}\text { S8/S9/Construcción del } \\
\text { número: Estrategias de } \\
\text { conteo. }\end{array}$ & Estudio de caso: "Enumeración". \\
\hline $\begin{array}{l}\text { S10/Resolución de problemas } \\
\text { en Educación Parvularia. }\end{array}$ & $\begin{array}{l}\text { Estudio de clase: Planificación de experiencia sobre la enseñanza } \\
\text { del número para aplicar con párvulos. }\end{array}$ \\
\hline $\begin{array}{l}\text { S11/S12/Construcción del } \\
\text { Número. }\end{array}$ & Estudio de caso: Enseñanza del "10". \\
\hline $\begin{array}{l}\text { S13/Construcción del } \\
\text { Número: Representaciones } \\
\text { del Objeto Matemático. }\end{array}$ & $\begin{array}{l}\text { Análisis de video. Estudio de clase: Análisis de representaciones } \\
\text { del objeto matemático incluidas en la planificación de la experiencia } \\
\text { a implementar con párvulos. }\end{array}$ \\
\hline $\begin{array}{l}\text { S14/Resolución de problemas } \\
\text { en la Educación Parvularia }\end{array}$ & $\begin{array}{l}\text { Estudio de clase: Análisis de video de experiencia aplicada en aula } \\
\text { para su mejora. }\end{array}$ \\
\hline
\end{tabular}

Fuente: elaboración propia.

A partir de lo expuesto, el objetivo de la investigación fue estudiar el efecto del curso de didáctica de la matemática en el conocimiento para la enseñanza del número en educadoras de párvulos en formación inicial docente ${ }^{1}$.

\section{MÉTODO}

\subsection{DISEÑO}

Se realizó una investigación con enfoque mixto, diseño cuasi-experimental complementado con técnicas cualitativas. El diseño intrasujeto simple, con medición antes y después

La investigación es parte del Proyecto FONDECYT 1111009 “Taller de didáctica de la matemática: una actividad curricular innovadora para la formación de profesores básicos y educadoras de párvulos. Validación de constructos y herramientas para la formación inicial docente". 
de una intervención, resulta ventajoso debido al control del error, el mismo individuo se convierte en criterio de control extrayendo la varianza de error debida a las diferencias intersujetos, lo que favorece una mayor potencia estadística en el contraste (Balluerka y Vergara, 2001). Las técnicas cualitativas contemplaron entrevistas y el análisis de mapas conceptuales.

\subsection{PARTICIPANTES}

La muestra no probabilística estuvo compuesta por una cohorte de 39 estudiantes, mujeres, edad media 22 años, de tercer año de la carrera de Educación Parvularia de una universidad chilena, que participaron en el año 2011 en el curso de didáctica de la matemática. Las mismas participantes constituyeron la unidad de estudio para las técnicas cualitativas.

\subsection{INTERVENCIÓN}

El curso de didáctica de la matemática se implementó durante el segundo semestre del año 2011 con 16 sesiones semanales de 90 minutos. Estuvo a cargo de una profesora de Educación Superior, con estudios y experiencia en formación inicial docente y didáctica de la matemática para Educación Parvularia. Paralelo al curso, las estudiantes se encontraban cursando una asignatura de práctica intermedia en un nivel de Transición II en el que implementaron y videograbaron una actividad de enseñanza del número.

\subsection{INSTRUMENTOS}

Para el estudio cuantitativo se utilizó un test de conocimiento y para el estudio cualitativo se utilizaron entrevistas y mapas conceptuales.

- Test de conocimiento para la enseñanza del número en estudiantes de Educación Parvularia. El instrumento presenta dos situaciones de aula de Educación Parvularia, una de lógica y otra de número, sobre las cuales se plantean ítems sobre conceptos matemáticos, organización de la enseñanza y etapas del desarrollo del pensamiento lógico matemático. La fiabilidad del test es de 0,72, considerada aceptable (Nunally y Bernstein, 1995), con 0,61 para la componente CC, 0,50 para CPC-CRAC y 0,52 para CPC-Ens. El test fue validado en un estudio previo (Goldrine et al., en revisión). El instrumento fue respondido como pre y postest por 35 estudiantes.

- Mapas conceptuales. Se solicitó a las estudiantes elaborar un mapa conceptual sobre la enseñanza del número en Educación Parvularia, con la indicación de responder ¿qué enseñar?, ¿cómo enseñar?, ¿a qué edad? y ¿con qué enseñar el concepto de número? 28 estudiantes elaboraron mapas al inicio y al término del curso.

- Entrevista sobre la práctica docente. Las entrevistas se aplicaron a 23 estudiantes al término del curso. Cada entrevista se inició observando el video de la experiencia de enseñanzaaprendizaje del número que la estudiante desarrolló con un grupo de párvulos. Para cada entrevista se seleccionaron extractos del video de la estudiante. El protocolo de la entrevista incluyó preguntas sobre los objetivos, planificación e implementación de la actividad en el aula, y sobre la percepción de la estudiante acerca de su capacidad de enseñanza del número antes y después del curso de didáctica. 


\subsection{PROCEDIMIENTO DE ANÁLISIS}

Los resultados del test se sometieron a un análisis descriptivo y, complementariamente, se utilizaron gráficos de cajas y de curvas de densidad de núcleo para observar el comportamiento de los resultados antes y después de la intervención. Se efectuó un análisis estadístico paramétrico cuando se cumplían los supuestos requeridos.

Los mapas conceptuales se evaluaron mediante una rúbrica que consignó la presencia de conceptos principales y subordinados de las componentes CC, CPC-Ens y CPC-CRAC, conexiones entre conceptos y estructura global del mapa, con puntaje mínimo de 0 y máximo de 18 puntos. Se compararon los puntajes de los mapas elaborados antes y después del curso. Los mapas fueron revisados por dos investigadoras en forma separada, con una fiabilidad interjueces de $75 \%$, asignando el puntaje final por consenso.

Las transcripciones de las entrevistas se sometieron a un análisis interpretativo para establecer categorías con respecto a los conocimientos reportados por las estudiantes. Las transcripciones fueron revisadas por dos investigadoras por separado para identificar ideas recurrentes y establecer categorías iniciales que luego se reagruparon en categorías mayores (Martínez, 1998). Se sumó una tercera investigadora para concordar las categorías definitivas y seleccionar extractos representativos.

\section{RESULTADOS}

A nivel cuantitativo se presentan los resultados del test y a nivel cualitativo los análisis de los mapas conceptuales y entrevistas.

\subsection{ESTUDIO CUANTITATIVO}

Los resultados del test, antes y después del curso, muestran diferencias en el conocimiento para la enseñanza del número en las futuras educadoras de párvulos. Véase la Figura $\mathrm{N}^{\circ} 1$.

Figura $N^{\circ} 1$. Resultados pre y post test por componente

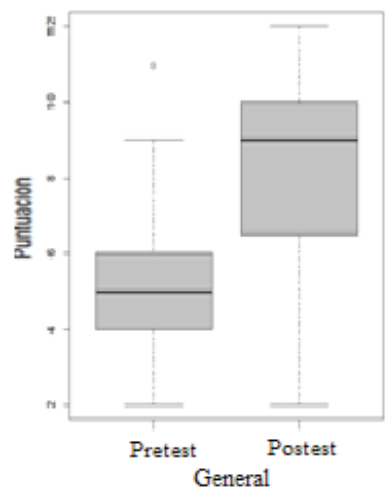

Fuente: elaboración propia.

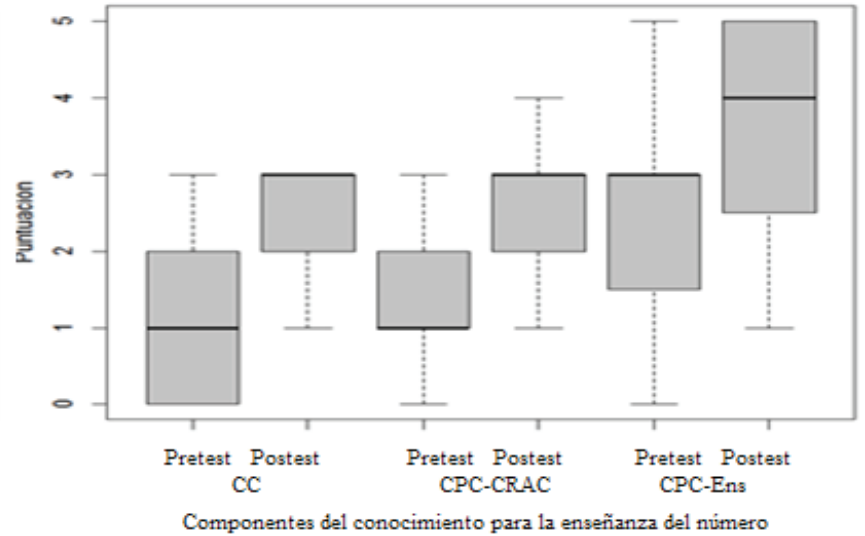

Componentes del conocimiento para la enseñanza del número 
En CC, la mediana en el pretest se sitúa en 1 y en el postest se posiciona en 3. La dispersión es mayor en el pretest, indicando que los resultados del postest fueron algo más homogéneos y que la mejora en el desempeño involucró a la mayoría de las estudiantes. En CPC-CRAC, la mediana del pretest es igual a 1 y el postest aumenta a 3, con similar dispersión. En CPC-Ens, la mediana del pretest es 3 y la del postest es 4, disminuyendo la dispersión y mostrando mejoras en el desempeño de las estudiantes.

Las curvas de densidad de núcleo ilustran con mayor claridad la distribución de la variabilidad y las diferencias entre puntuaciones para las componentes (Figura $\mathrm{N}^{\circ} 2$ ). Cabe destacar que en todas las componentes se observan diferencias a favor del postest. Sin embargo, las dispersiones son altas, en particular destaca la gran dispersión en el caso del pretest de la componente CC.

Figura $N^{\circ}$ 2. Curvas de densidad de núcleo de puntuaciones por componente antes (línea continua) y después (línea discontinua) del curso

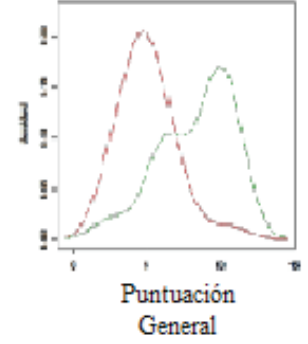

Fuente: elaboración propia.

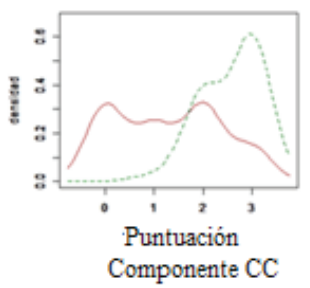

Componente CC
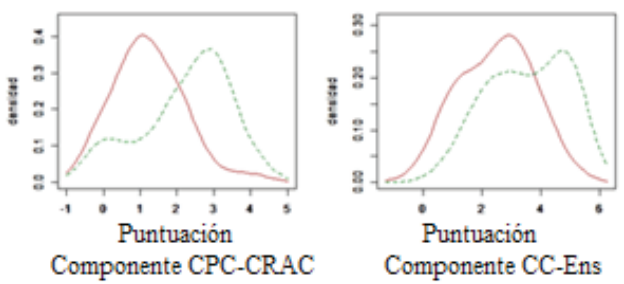

Utilizando el análisis de varianza mixto para los contrastes que cumplieron con los supuestos para contraste paramétrico, se obtienen resultados significativos con diferencias entre pre y post en CC, CPC-CRAC y CPC-Ens. La Tabla $\mathrm{N}^{\circ} 2$ muestra los resultados de CPC-CRAC y CPC-Ens.

Tabla $N^{\circ}$ 2. ANOVA mixto sobre las condiciones de las variables CPC-CRAC y CPC-Ens

\begin{tabular}{|c|c|c|c|c|c|}
\hline Variable (pretest - postest) & Gl & Suma cuadrados & Media cuadrática & Razón F & Valor $\mathrm{p}$ \\
\hline CRA & 1 & 28.93 & 28.929 & 45.6 & 0.0000 \\
\hline Residuos & 34 & 21.57 & 0.634 & & \\
\hline ENS & 1 & 19.56 & 19.557 & 25.63 & 0.0000 \\
\hline Residuos & 34 & 25.94 & 0.763 & & \\
\hline
\end{tabular}

Fuente: elaboración propia.

En la Tabla $\mathrm{N}^{\circ} 3$ se muestra la prueba Friedman con Ji-cuadrado con los resultados para la componente $\mathrm{CC}$ y el resultado general. Como se observa, también se presentan 
diferencias estadísticamente significativas entre el pretest y el postest en la componente CC y en los resultados de la prueba completa.

Tabla $N^{\circ}$ 3. Prueba de Friedman sobre la variable CC y resultado general

\begin{tabular}{|c|c|c|c|}
\hline Variable (pretest - postest) & Ji-cuadrado & Gl & Valor $p$ \\
\hline Componente CC & 70 & 1 & 0.0000 \\
\hline General & 16.3333 & 1 & 0.0000 \\
\hline
\end{tabular}

Fuente: elaboración propia.

\subsection{ESTUDIO CUALITATIVO}

\subsubsection{Mapas conceptuales}

La Tabla $\mathrm{N}^{\circ} 4$ presenta los puntajes obtenidos por las futuras educadoras en los mapas conceptuales al inicio y al término del curso.

Tabla $N^{\circ}$ 4. Número de estudiantes según puntaje en mapas conceptuales al inicio y al término del curso

\begin{tabular}{|c|c|c|}
\hline $\begin{array}{c}\text { Rango } \\
\text { puntaje }\end{array}$ & $\begin{array}{c}\mathrm{N}^{\circ} \text { estudiantes } \\
\text { (inicio curso) }\end{array}$ & $\begin{array}{c}\mathrm{N}^{\mathrm{o}} \text { estudiantes } \\
\text { (término curso) }\end{array}$ \\
\hline $0-2$ & 1 & 0 \\
\hline $3-5$ & 12 & 6 \\
\hline $6-8$ & 12 & 4 \\
\hline $9-11$ & 3 & 8 \\
\hline $12-16$ & 0 & 0 \\
\hline $17-18$ & & \\
\hline
\end{tabular}

Fuente: elaboración propia.

El puntaje mediano al inicio del curso fue de 6 puntos y el puntaje mediano al finalizar el curso fue de 10 puntos. Al inicio del curso, 25 de las 28 estudiantes obtuvieron un puntaje menor o igual a 8 puntos (89\%), y 3 estudiantes un puntaje mayor a $8(11 \%)$. Al término del curso, 10 estudiantes obtuvieron un puntaje menor o igual a 8 puntos (40\%), 18 estudiantes obtuvieron un puntaje mayor o igual a 8 puntos, equivalente a un $60 \%$, mostrando diferencias a favor del curso. 
La Figura $\mathrm{N}^{\circ} 3$ muestra el mapa de una estudiante que obtuvo alto puntaje al término del curso (EI: mapa de la Estudiante al Inicio del curso. ET: mapa de la Estudiante al Término del curso).

Figura $N^{\circ}$ 3. Mapas de una estudiante al inicio y término del curso.
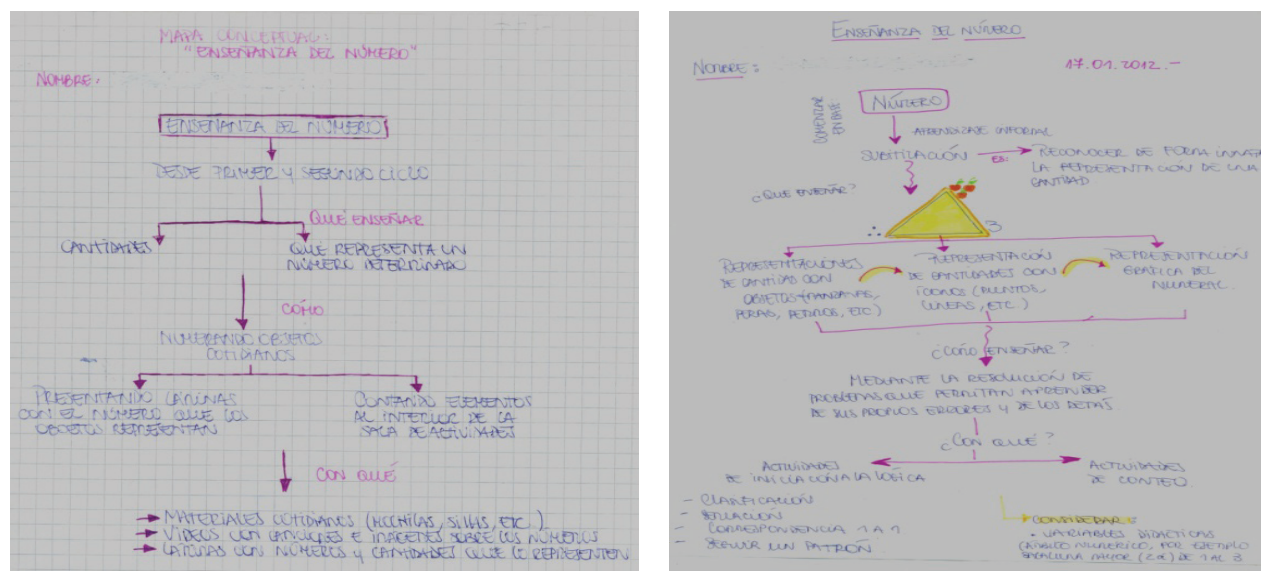

Fuente: elaboración propia.

El mapa ET (16 puntos) en comparación con el mapa EI (6 puntos) muestra diferencias a favor del curso en el caso de una estudiante en particular. Con respecto a qué enseñar, la estudiante consigna "iniciación a la lógica y actividades de conteo", "subitización" y muestra tres tipos de representación de cantidad: pictográfica, icónica y simbólica, con un dibujo que ilustra esta idea. Sobre cómo enseñar, la estudiante consigna "mediante la resolución de problemas que permitan aprender de sus propios errores y de los demás" y menciona "variables didácticas". Tras el análisis de la totalidad de los mapas, se destaca que algunos mapas incluyeron nociones de lógica, como clasificación, seriación y correspondencia; y nociones de número como ordinalidad, cardinalidad y principios de conteo, aportando evidencia del CC alcanzado por las estudiantes al término del curso. En relación al CPC-Ens, algunas estudiantes mencionan el enfoque de resolución de problemas como estrategia a considerar en la organización de la enseñanza del número. En cuanto al CPC-CRAC, algunas estudiantes indican la regulación del ámbito numérico según la edad de los niños.

\subsubsection{Entrevistas}

El análisis de las entrevistas arrojó seis categorías: objetivos de la actividad desarrollada con un grupo de párvulos, valoración de la mediación docente, planificación de la actividad, presencia de una situación problema, concepción de la enseñanza de la matemática y opinión con respecto al curso. A continuación se presentan estas categorías con extractos de las entrevistas. 
Con respecto al objetivo de la actividad, las estudiantes señalaron: promover el conteo (17 estudiantes), estrategias de conteo (6), y representación de cantidades (6). Se muestran algunos extractos de una entrevista que muestra que el propósito de la actividad fue la representación de cantidades: "Tener nociones (...) de las cantidades y cómo lo iban a representar, (...) algunos [niños], hicieron los botones, los circulitos y otros inmediatamente hicieron también el número" (Estudiante 6, E6). Nueve estudiantes logran identificar en las acciones de los niños la presencia de los principios de conteo de Gelman \& Gallistel (1978). Por ejemplo: "El principio de término a término lo ocuparon harto, pero también utilizaban los de abstracción y de cardinalidad, el del orden estable, ese lo tienen más que aprendido con la serie oral, sabían contar perfectamente hasta veinte" (E12).

Con respecto a la valoración de la mediación docente (preguntas, intervenciones y/o apoyos), las respuestas de las estudiantes se agruparon en categorías: adecuadas, parcialmente adecuadas y no adecuadas. Doce estudiantes valoraron su mediación como adecuada, argumentando que usaron preguntas en la interacción con los párvulos para apoyar la resolución del problema. Por ejemplo: "en todo momento traté de hacerles una devolución, cuando los niños preguntaban o tenían alguna dificultad yo siempre les hablaba, les hacía preguntas para que ellos resolvieran el problema" (E1). Nueve estudiantes consideran que su mediación no fue adecuada porque les dieron las respuestas a los párvulos. Por ejemplo: "me impuse cuando le dije: ipero tú ocupa un dedo! [para contar]. Entonces ésas son las cosas que de repente uno dice jno!, la idea es que ellos solitos se vayan dando cuenta" (E2). Dos de las estudiantes consideran que su mediación fue parcialmente adecuada pero se puede mejorar. Por ejemplo: "pude haber implementado otra pregunta o haber seguido insistiendo hasta que el niño lo lograra” (E10).

Las estudiantes mencionan que incluyeron en la planificación elementos que antes del curso no consideraban al planificar actividades de matemática: una situación problema (13 estudiantes), elementos del análisis a priori como institucionalización, aprendizajes previos, conocimientos en juego y devolución (8 estudiantes), pregunta central (7 estudiantes), cambios en la mediación hacia una devolución que no entregue las respuestas a los niños (6 estudiantes) y material concreto (5 estudiantes). Como se desprende de los siguientes extractos: "Estaba acostumbrada a ver que los niños contaran por contar sin un objetivo claro, sin mayores desafios" (E12)". "Dar la pregunta como fuente de la situación (problema) (E14)".

La mayoría de las estudiantes (16 de 23) considera que la actividad realizada planteaba una situación problema a los párvulos; tres estudiantes consideran que la actividad constituyó un problema sólo para algunos de los niños del grupo; y dos estudiantes consideran que la actividad no fue un problema para el grupo. Por ejemplo: "Yo creo que sí, porque fue desafiante y porque sus conocimientos previos no les permitían llegar y contar como lo habían hecho siempre" (E12). "Creo que esta actividad en particular tal vez no, porque fue el ámbito numérico muy bajo, si lo hubiéramos hecho con un ámbito numérico mayor, a lo mejor hubiera sido un desafio" (E5).

Para las estudiantes una situación problema genera un desafío (6), se buscan soluciones y/o incentiva el pensamiento divergente (6), y el aprendizaje es significativo (5). Por ejemplo: "Las actividades con que yo enseñaba matemáticas eran expositivas, yo les mostraba láminas, cuerpos geométricos, pero nunca les plantee a los niños un desafío" (E2); "sirve para que ellos puedan pensar, para que se las puedan como 'cranear' entre comillas, cómo buscar una solución y no que se les dé todo tan fácil" (E7); "es más significativo 
un problema que un ejercicio en una hoja” (E8); "los aprendizajes de las matemáticas se tienen que plantear con un problema porque siempre uno para resolver las matemáticas tiene que buscar estrategias" (E5); “creo que la resolución de problemas es el camino que nos puede llevar al aprendizaje de las matemáticas de manera más significativa” (E13).

En las entrevistas, las estudiantes señalaron cambios en su concepción sobre la enseñanza de las matemáticas después del curso de didáctica, afirman que los niños son constructores de sus aprendizajes y que las matemáticas deben ser enseñadas de manera lúdica (10 estudiantes), se deben considerar los aprendizajes previos de los niños y realizar actividades a través de secuencias didácticas (9 estudiantes), y que la enseñanza de las matemáticas debe ser a través de la resolución de problemas (4 estudiantes). Por ejemplo: "Los niños cuando hacen un proceso matemático, digamos, lo hacen propio, no es algo que uno les enseñe o les transmita, entonces el enseñar entre comillas o guiarlos para que ellos realicen ese proceso fue el cambio que yo hice" (E23). "Tomar en base lo que los niños traen de su hogar, como informal y ahí partir a lo formal" (E17). "Yo sé ahora que tengo que hacer actividades de resolución de problemas" (E11).

En cuanto a las diferencias en la enseñanza de la matemática después del curso, señalan cambios en el tipo de actividades, antes eran actividades expositivas y ahora son desafiantes (13), han notado cambios en su mediación (6), aprecian un aprendizaje personal llegando a sentirse más preparadas y a encontrarle sentido a las matemáticas (6). Por ejemplo: "Uno siempre tiende a llevar a los niños a la solución y decirles cómo contar y no dejar que ellos busquen su estrategia, uno le dice 'te ayudo a contar' y entonces uno cuenta, finalmente uno le hace la actividad al niño, pero ahora no, ahora uno se da cuenta y cuando los niños hacen preguntas, uno les hace preguntas abiertas y tiende siempre a hacer preguntas" (E5).

Las estudiantes consideran que el curso les dio herramientas para la implementación de planificaciones con un enfoque de resolución de problema (15), y para mejorar su mediación hacia una devolución que no entregue las respuestas a los niños (6). Por ejemplo: "Hay que tener mucho ojo con la mediación que uno está haciendo y no darle la respuesta” (E8).

Con respecto a la metodología del curso, las estudiantes señalan la vinculación de la teoría con la práctica (11). Por ejemplo: "como fortaleza creo que igual siempre se intentó combinar lo práctico con lo teórico” (E13).

\section{DISCUSIÓN}

Tanto los resultados del test, evidencia cuantitativa, como las entrevistas y los mapas conceptuales, evidencias cualitativas, muestran indicios de un posible efecto del curso en el conocimiento para la enseñanza del número de las futuras educadoras.

Las pruebas estadísticas mostraron una diferencia significativa. Las diferencias de los resultados del test al inicio y al término del curso estarían arrojando evidencia de la pertinencia del constructo y de las metodologías utilizadas en el curso. Las diferencias entre los pretest y los postest revelarían que el curso tuvo mayor efecto en la componente $\mathrm{CC}$, en comparación con las otras componentes. En la componente CC las estudiantes presentaron el más bajo puntaje al inicio del curso y obtuvieron el mayor avance. Estos resultados son provechosos en términos del conocimiento docente que las estudiantes lograron construir con el curso, ya que en opinión de algunos autores esta componente es base para el avance en las otras (Sliva, Strage \& Bergthold, 2007; Ball, Lubienski \& Mewborn, 2001; Ma, 1999). 
Para Ma (1999) el docente requiere de un profundo conocimiento de la matemática a enseñar, este conocimiento es clave en la configuración de las otras componentes del conocimiento docente y estaría asociado a la efectividad de la enseñanza. Llevado al ámbito de la formación de educadoras de párvulos, esto significa que las futuras docentes se verían favorecidas al desarrollar un conocimiento profundo de las nociones matemáticas que se abordan en el nivel de Educación Parvularia. En este sentido, el curso podría constituirse en un recurso curricular para la formación inicial docente.

Los resultados del análisis de los mapas también constituirían evidencia de las bondades del curso. Como instrumento de indagación, el mapa conceptual permitió a las estudiantes comunicar su conocimiento antes y después del curso, y por tanto, las ideas expuestas en los mapas se pueden asociar al conocimiento que las estudiantes construyeron durante el curso. La mayor parte de las nociones matemáticas y didácticas explicitadas por las estudiantes al final del curso no estaban presentes en los mapas conceptuales elaborados al inicio del curso.

Los resultados del análisis de las entrevistas también aportarían evidencias en favor del curso. Las entrevistas al término del curso permiten identificar la presencia de las componentes del constructo conocimiento docente para la enseñanza del número. En sus discursos, las estudiantes utilizan apropiadamente nociones matemáticas trabajadas en el curso como cardinalidad, ordinalidad, representación de cantidad, principios de conteo, serie numérica, operaciones matemáticas y resolución de problemas. El uso de este lenguaje puede ser interpretado como enriquecimiento del conocimiento docente, particularmente en CC. En lo que respecta a la gestión de la enseñanza en el aula, varias estudiantes enfatizaron que intentaron promover la resolución de problemas y realizar una mediación -en base a preguntas- que evita entregar a los párvulos las estrategias y respuestas al problema. Esta mediación concretiza la puesta en acción del concepto de devolución ${ }^{2}$ y puede interpretarse como un CPC-Ens. La formulación de preguntas por las futuras educadoras a los párvulos durante las actividades constituye una experiencia docente de seguimiento al razonamiento infantil, y en este sentido, puede interpretarse como un indicio de la comprensión del pensamiento del niño que evidencia CPC-CRAC en las estudiantes.

Conforme al diseño del estudio, sobre la base de una intervención, las metodologías de análisis de videos de clases, el estudio de casos y el estudio de clases sustentarían la potencialidad del curso, en virtud de su adaptabilidad para integrar teoría y práctica y favorecer la reflexión docente. Varias estudiantes en las entrevistas señalaron que las metodologías del curso favorecieron la vinculación de la teoría con la práctica en aula, y que el curso les brindó herramientas para implementar el enfoque de resolución de problemas y mejorar la mediación en la enseñanza de la matemática. El discurso de las estudiantes en las entrevistas constituiría evidencia de la reflexión docente que se puso en juego durante las clases llevadas adelante a través de estas metodologías (ver Tabla $\mathrm{N}^{\circ} 1$ ). En los casos estudiados, sesiones 8, 9, 11 y 12, la discusión en torno a los conflictos pedagógicos y didácticos habría motivado la reflexión docente y el análisis a partir de los referentes conceptuales revisados en el curso. Con respecto al análisis de videos, en las sesiones

El proceso devolución pone en juego la interacción del alumno con el problema y del alumno con el profesor respecto al problema. El profesor, a través de preguntas en relación con el conocimiento previsto, lleva el proceso de enseñanza de modo de favorecer los aprendizajes. De esta manera, el proceso de devolución plantea una enseñanza centrada en la actividad del alumno (Olfos, Guzmán y Estrella, 2014). 
1, 3, 5, 6 y 13, las estudiantes observaron videos y comentaron aspectos como la tarea matemática, la devolución docente y los desempeños matemáticos infantiles, extrayendo reflexiones que estarían nutriendo una visión crítica de las propias prácticas docentes. En cuanto al estudio de clases, en la sesión 10, las estudiantes planificaron en grupos una experiencia de enseñanza del número para un grupo de párvulos que implementaron y videograbaron. Luego, en la sesión 14, las estudiantes observaron los videos, reflexionaron sobre las fortalezas y las falencias de su enseñanza, y propusieron mejoras, poniendo en juego los contenidos tratados en el curso.

\section{CONCLUSIONES}

La principal conclusión del estudio es de carácter inductiva: el nivel de éxito en el curso de didáctica de la matemática estaría asociado al nivel de conocimiento para la enseñanza del número alcanzado por las futuras educadoras de párvulos. Sin embargo, es importante tener presente que una limitación de este estudio es su diseño cuasi experimental, por lo que la asociación entre la implementación de las metodologías del curso y el conocimiento docente adquirido por las futuras educadoras de párvulos no sería necesariamente de tipo causal.

Los autores postulan que la efectividad del curso podría estar asociada a la relación dialéctica que se establece entre el constructo de conocimiento docente que fundamenta el curso, y las tres metodologías de enseñanza que integran teoría y práctica. Estas metodologías, como se desprende de la literatura revisada, estarían promoviendo la reflexión docente y, a través de ésta, la construcción de conocimiento docente en la futura educadora de párvulos.

Un aspecto relevante de la investigación es que dio indicios de la efectividad de un curso centrado en un constructo sobre el saber que requiere el futuro profesor para aprender a enseñar. Este constructo está fundamentado en la literatura actual sobre el conocimiento del profesor (McCray \& Chen, 2012; Lee, 2010; Hill, Ball \& Schilling, 2008; Krauss, Baumert \& Blum, 2008; McCray, 2008; Platas, 2008; Fennema \& Franke, 1992; Goldrine et al., en revisión). El curso integra el conocimiento del contenido a enseñar, $\mathrm{CC}$, con el conocimiento de la enseñanza del contenido, CPC-Ens, y el conocimiento de la relación del alumno con el saber, CPC- CRAC. El curso logró operacionalizar la propuesta de Shulman (1987) y desarrollos teóricos posteriores en el contexto de la formación inicial de educadoras para la enseñanza del número.

La reflexión docente generada por las metodologías del curso -estudio de clases, estudio de casos y análisis de videos-, podría estar movilizando en las futuras educadoras la vinculación de las componentes CC, CPC-Ens y CPC-CRAC, fortaleciendo la construcción del conocimiento para enseñar. De este modo, el curso -objeto de la investigaciónconstituiría, tanto a nivel de constructo, contenidos y metodologías, un recurso curricular innovador para las carreras de Educación Parvularia.

La validez de la investigación se atribuiría a las evidencias entregadas por el enfoque mixto utilizado, que triangula los resultados cuantitativos del test con las evidencias cualitativas obtenidas a partir de entrevistas y mapas conceptuales. La validez del estudio cuantitativo reside en el uso del "Test de conocimiento para la enseñanza del número en estudiantes de Educación Parvularia" validado previamente, y que mide las componentes del constructo de conocimiento docente que fundamenta el curso. 
La investigación se centró en el análisis de las diferencias en el conocimiento de las futuras educadoras entre el inicio y el término del curso, identificando las evidencias de los resultados del curso. Sería conveniente continuar enriqueciendo el estudio con evidencias de proceso, a través del análisis de la implementación de las tres metodologías que habrían favorecido la construcción de conocimiento a partir de la reflexión de las futuras educadoras.

Junto a lo anterior, se sugiere continuar estudiando metodologías formativas que tiendan un puente entre el conocimiento de la enseñanza de la matemática que aprende la futura educadora a nivel declarativo, y el conocimiento que despliega en la enseñanza en el aula. Constituye un desafío para la formación docente lograr implementar un sistema de andamiaje que permita a la futura educadora transitar desde un aprendizaje declarativo de la enseñanza de la matemática, hacia prácticas efectivas que impacten en los aprendizajes de los párvulos. Futuras investigaciones podrían relacionar el conocimiento para la enseñanza del número con las prácticas de aula de las futuras educadoras, y el impacto de este conocimiento en el aprendizaje del número en los párvulos.

\section{REFERENCIAS BIBLIOGRÁFICAS}

Ávalos, B. (2011). Teacher Professional Development in Teaching and Teacher Education over Ten Years. Teaching and Teacher Education, vol.27 (1), 10-20.

Ball, D., Lubienski, S. \& Mewborn, D. (2001). Research on Teaching Mathematics: The Unsolved Problem of Teachers' Mathematical Knowledge. In V. Richardson (Ed.), Handbook of Research on Teaching. $4^{\text {th }}$ Edition (pp. 433- 456). Washington, DC: American Educational Research Association.

Balluerka, N. y Vergara, A. (2002). Diseños de investigación experimental en psicología. Madrid: Prentice Hall.

Borko, H., Jacobs, J., Eiteljorg, E. \& Pittman, M. (2008). Video as a Tool for Fostering Productive Discussions in Mathematics Professional Development. Teaching and Teacher Education, vol.24 (2), 417-436.

Brophy, J. (Ed.). (2004). Using Video in Teacher Education. The Netherlands: Elsevier.

Darling-Hammond, L. (1997). Doing What Matters Most: Investing in Quality Teaching. New York: National Commission on Teaching \& America's Future.

Esen, Y., Özgeldi, M. \& Haser, Ç. (2012). Exploring Pre-service Early Childhood Teachers' Pedagogical Content Knowledge for Teaching Mathematics. 12th International Congress on Mathematical Education. COEX, Seoul, Korea.

Fennema, E. \& Franke, M. (1992). Teachers' Knowledge and its Impact. In D. A. Grouws (Ed.), Handbook of Research on Mathematics Teaching and Learning (pp. 147-164). New York: MacMillan Publishing.

Friz, M., Sanhueza, S., Sánchez, A., Sámuel, M. y Carrera, C. (2009). Concepciones en la enseñanza de la matemática en educación infantil. Perfiles Educativos, vol.31 (125), 62-76.

(2008). Conocimiento de las competencias profesionales implicadas en la enseñanza de las matemáticas en Educación Preescolar. Segundo Encuentro de Educación Inicial. Pontificia Universidad Católica de Chile, Santiago.

Gellman, R. \& Gallistel, C. R. (1978). The Child's Understanding of Number. Harvard: Harvard University Press.

Goldrine, T., Estrella, S., Olfos, R. y Cáceres P. (en revisión). Diseño y estudio de validez de una prueba de conocimientos para la enseñanza del número en futuras maestras de educación infantil. Manuscrito sometido a publicación.

Hammerness, K., Darling-Hammond, L., Bransford, J., Berliner, D., Cochran-Smith, M., 
Mc Donald, M. \& Ziechner, K. (2005). How Teachers Learn and Develop. In K. Hammerness, L. Darling-Hammond \& J. Bransford (Eds.), Preparing Teachers for a Changing World (pp. 358-389). San Francisco: Jossey-Bass.

Harford, J. \& MacRuairc, G. (2008). Engaging Student Teachers in Meaningful Reflective Practice. Teaching and Teacher Education, vol.24 (7), 1884-1892.

Hiebert, J., Carpenter, T. P., Fennema, E., Fuson, K. C., Wearne, D. \& Murray, H. (1997). Making Sense: Teaching and Learning Mathematics with Understanding. Portsmouth, NH: Heinemann Publications.

Hill, H., Ball, D. \& Schilling, S. (2008). Unpacking Pedagogical Content Knowledge, Conceptualizing and Measuring Teachers' Topic Specific Knowledge of Students. Journal for Research in Mathematics Education, vol.39 (4), 327-400.

Isoda, M. y Olfos, R. (2009). El enfoque de resolución de problemas en la enseñanza de la matemática a partir del estudio de clases. Valparaíso: Ediciones Universitarias de Valparaíso.

., Arcavi, A. y Mena, A. (2007). El estudio de clases japonés en matemáticas: Su importancia para el mejoramiento de los aprendizajes en el escenario global. Valparaíso: Ediciones Universitarias de Valparaíso.

Koc, Y., Peker, D. \& Osmanoglu, A. (2009). Supporting Teacher Professional Development through Online Video Case Study Discussions: An Assemblage of Pre-service and In-service Teachers and the Case Teacher. Teaching and Teacher Education, vol.25 (8), 1158-1168.

Krauss, S., Baumert, J. \& Blum, W. (2008). Secondary Mathematics Teachers' Pedagogical Content Knowledge and Content Knowledge: Validation of the COACTIV Constructs. ZDM Mathematics Education, vol.40 (5), 873-892.

, Brunner, M. \& Kunter, M. (2008). Pedagogical Content Knowledge and Content

Knowledge of Secondary Mathematics Teachers. Journal of Educational Psychology, vol.100 (3), 716-725.

Lee, J. F. (2010). Exploring Kindergarten Teachers' Pedagogical Content Knowledge of Mathematics. International Journal of Early Childhood, vol.42 (1), 27-41.

(2008). A Hong Kong Case of Lesson Study - Benefits and Concerns. Teaching and

Teacher Education, vol.24 (5), 1115-1124.

Lundeberg, M., Levin, B. \& Harrington, H. (1999). Who Learns What from Cases and How?

The Research Base for Teaching and Learning with Cases. Mahwah: Lawrence Erlbaum Associates.

Ma, L. (1999). Knowing and Teaching Elementary Mathematics. Teachers' Understanding of Fundamental Mathematics in China and the United States. Mahwah: Erlbaum.

Martínez, M. (1998). La investigación cualitativa etnográfica en educación. México D.F.: Trillas.

McCray, J. \& Chen, J. (2012). Pedagogical Content Knowledge for Preschool Mathematics: Construct Validity of a New Teacher Interview. Journal of Research in Childhood Education, vol.26 (3), 291-307.

(2008). Pedagogical Content Knowledge for Preschool Mathematics: Relationships to Teaching Practices and Child Outcomes. PhD Thesis. Erikson Institute, Loyola University Chicago.

MINEDUC (2012). Informe Evaluación Inicia 2011. Recuperado desde http://www. evaluacioninicia.cl

(2011). Informe Evaluación Inicia 2010. Recuperado desde http://www.evaluacioninicia.cl (2010). Evaluación Diagnóstica Inicia. Resultados Aplicación 2009. Recuperado desde http://www.educarchile.cl/UserFiles/P0001/File/CR_Articulos/RESULTADOS_inicia_2009.pdf

Morales, S., Quilaqueo, D. y Uribe, P. (2010). Saber pedagógico y disciplinario del educador de infancia. Un estudio en el sur de Chile. Perfiles Educativos, vol.32 (130), 49-66.

Murillo, J. (2006). La formación de docentes: Una clave para la mejora educativa. En M. Robalino y A. Körner (Coords.), Modelos innovadores en la formación inicial docente (pp. 11-18). Santiago: UNESCO. 
Nunally, J. y Bernstein, I. (1995). Teoría psicométrica. México D. F.: McGraw-Hill.

Olfos, R., Guzmán, I. y Estrella, S. (2014). Gestión didáctica en clases y su relación con las decisiones del profesor: El caso del Teorema de Pitágoras en séptimo grado. Boletim de Educação Matemática, vol.28 (48), 341-359.

Perrenoud, P. (2004). Desarrollar la práctica reflexiva en el oficio de enseñar. Barcelona: Graò.

Platas, L. M. (2008). Measuring Teachers' Knowledge of Early Mathematical Development and their Beliefs about Mathematics Teaching and Learning in the Preschool Classroom. PhD Thesis. University of California, Berkeley.

Puchner, L. D. \& Taylor, A. R. (2006). Lesson Study, Collaboration and Teacher Efficacy: Stories from Two School-based Math Lesson Study Groups. Teaching and Teacher Education, vol.22 (7), 922-934.

Reyes, C. (2011). Estudio de casos en la formación de profesores de matemáticas: Integrando matemáticas y pedagogía. Santiago: J.C. Sáez.

Santagata, R., Zannoni, C. \& Stigler, W. (2007). The Role of Lesson Analysis in Pre-service Teacher Education: An Empirical Investigation of Teacher Learning from a Virtual Video-based Field Experience. Journal of Mathematics Teacher Education, vol.10 (2), 123-140.

Saracho, O. \& Spodek, B. (2007). Contemporary Perspectives on Socialization and Social Development in Early Childhood Education. Charlotte: IAP Publishing.

Seidel, T., Stürmer, K., Blomberg, G., Kobarg, M. \& Schwindt, K. (2011). Teacher Learning from Analysis of Videotaped Classroom Situations: Does it make a Difference whether Teachers observe their Own Teaching or that of others? Teaching and Teacher Education, vol.27 (2), 259-267.

Shulman, L. (1987). Knowledge and Teaching: Foundations of the New Reform. Harvard Educational Review, vol.57 (1), 1-22.

Schmidt, W. H., Tatto, M. T., Bankov, K., Blömeke, S., Cedillo, T., Cogan, L., Han, S. I., Houang, R. \& Schwille, J. (2007). The Preparation Gap: Teacher Education for Middle School Mathematics in Six Countries. Mathematics Teaching in the 21st Century (MT21). East Lansing, MI: MSU. Retrieved from http://usteds.msu.edu/related_research.asp

Sliva, J., Strage, A. \& Bergthold, T. (2007). Content Knowledge Achievement: Placing Mathematics Content for Prospective K-8 School Teachers in Context through Field Experiences. In T. Lamberg \& L. R. Wiest (Eds.), Proceedings of the 29th Annual Meeting of the North American Chapter of the International Group for the Psychology of Mathematics Education. University of Nevada, Reno.

Varol, F., Farran, D., Bilbrey, C., Vorhaus, E. \& Hofer, K. (2012). Professional Development for Preschool Teachers: Evidence for Practice. NHSA Dialog, vol.15 (1), 122-126. 
